\title{
Classes of Graphs Which Approximate the Complete Euclidean Graph
}

\author{
J. Mark Keil and Carl A. Gutwin \\ Department of Computational Science, University of Saskatchewan, \\ Saskatoon, Canada S7N 0W0
}

\begin{abstract}
Let $S$ be a set of $N$ points in the Euclidean plane, and let $d(p, q)$ be the Euclidean distance between points $p$ and $q$ in $S$. Let $G(S)$ be a Euclidean graph based on $S$ and let $G(p, q)$ be the length of the shortest path in $G(S)$ between $p$ and $q$. We say a Euclidean graph $G(S) t$-approximates the complete Euclidean graph if, for every $p, q \in S, G(p, q) / d(p, q) \leq t$. In this paper we present two classes of graphs which closely approximate the complete Euclidean graph. We first consider the graph of the Delaunay triangulation of $S, \operatorname{DT}(S)$. We show that $\mathrm{DT}(S)(2 \pi /(3 \cos (\pi / 6)) \approx 2.42)$ approximates the complete Euclidean graph. Secondly, we define $\theta(S)$, the fixed-angle $\theta$-graph (a type of geometric neighbor graph) and show that $\theta(S)$ $((1 / \cos \theta)(1 /(1-\tan \theta)))$-approximates the complete Euclidean graph.
\end{abstract}

\section{Introduction}

Let $S$ be a set of $N$ points in the Euclidean plane. Then a Euclidean graph $G(S)$ on $S$ has the property that each vertex corresponds to a point in $S$ and the weight of an edge is equal to the straightline Euclidean distance between the points it connects [7]. Euclidean graphs have been used as a modeling tool; for example, in circuit layout [7]. They also arise indirectly in the solution to geometric problems such as motion planning [2].

Let $d(p, q)$ be the Euclidean distance between points $p$ and $q$ and let $G(p, q)$ be the length of the shortest path in $G(S)$ between $p$ and $q$. We say a Euclidean graph $G(S) t$-approximates the complete Euclidean graph if, for every $p, q \in S$, $G(p, q) / d(p, q) \leq t$.

Approximating the complete Euclidean graph has potential application in the development of approximation algorithms or heuristics for problems that involve finding shortest distances in the plane [2], [4], [7]. The efficiency of the resulting algorithms would be dependent upon the number of edges in the approximating 
graph. For this reason, it is desirable to identify classes of graphs which closely approximate the complete Euclidean graph, yet contain only a linear number of edges.

The first known result of this type concerns the graph of the Delaunay triangulation in the $L_{1}$ metric, $\mathrm{DT}_{1}$. Chew [2] has shown that $\mathrm{DT}_{1}(S) \sqrt{10}$ approximates the complete Euclidean graph, independent of $S$ and $N$. Chew has also shown that the graph of the Delaunay triangulation based on an equilateral triangle for a unit "circle" 2-approximates the complete Euclidean graph [3]. Along similar lines Dobkin et al. [4] have shown that if DT(S) is the graph of the Delaunay triangulation with the Euclidean metric, $\operatorname{DT}(S)(((1+\sqrt{5}) / 2) \pi \cong 5.08)$ approximates the complete Euclidean graph, again independent of $S$ and $N$. Chew [2] was able to exhibit an example for which the ratio, of the shortest distance in $\mathrm{DT}(S)$ to the Euclidean distance, approaches $\pi / 2 \cong 1.57$. Thus the Delaunay triangulation $t$-approximates the complete Euclidean graph, where the lowest possible value of $t$ lies in the interval between 1.57 and 5.08. In Section 2 of this paper we show that the Delaunay triangulation indeed $(2 \pi /(3 \cos (\pi / 6)) \cong 2.42)$ approximates the complete Euclidean graph, independent of $S$ and $N$.

The Delaunay triangulations are good candidates for approximating graphs, since they contain only a linear number of edges and can be computed from the point set in $O(N \log N)$ time [6]. In some applications, however, it may be that the Delaunay approximates are not sufficiently close. In Section 3 we define the class of $\theta$-graphs and show that they can be computed from the point set in $O(N \log N)$ time. Each point in a $\theta$-graph defines an edge to a near neighbor in up to $k$ different directions, where $\theta=2 \pi / k$, thus a $\theta$-graph contains only a linear number of edges if $k$ is a constant. In Section 4 we show that, for $k>8$, a $\theta$-graph $((1 / \cos \theta)(1 /(1-\tan \theta)))$-approximates the complete Euclidean graph, again independent of both $S$ and $N$.

It should be noted that a Euclidean graph which approximates the complete Euclidean graph is a specific example of a graph spanner. Peleg and Schaffer [5], define a $t$-spanner for a connected simple graph, $G=(V, E)$, to be a subgraph $G^{\prime}=\left(V, E^{\prime}\right)$ such that, for every $u, v \in V$, $\operatorname{dist}\left(u, v, G^{\prime}\right) / \operatorname{dist}(u, v, G) \leq t$ where $\operatorname{dist}(u, v, H)$ denotes the distance from $u$ to $v$ in graph $H$. Graph spanners have applications in distributed systems and communications networks [5].

\section{Delaunay Triangulation}

In this section we consider how well the Delaunay triangulation approximates the complete Euclidean graph. We begin with some definitions. A Voronoi diagram of a set $S$ of points in the plane is a partition of the plane into regions, each corresponding to a point in $S$, such that, for a point $p \in S$, every point within its corresponding region is closer to $p$ than to any other point of $S$. By connecting two points of $S$ with an edge if and only if their corresponding Voronoi regions share a boundary, then a Euclidean graph results which is known as the Delaunay triangulation of $S$. This Voronoi dual is in fact a triangulation under the standard 
assumption that no four points of $S$ are cocircular [6]. Under this assumption, the Delaunay triangulation of $S$ can be alternatively defined as the Euclidean graph formed by joining a pair of points $p$ and $q$ if and only if there exists a circle passing through $p$ and $q$ that does not contain any point of $S$ in its interior.

For the remainder of this section we consider the shortest path in DT $(S)$ between two points $p$ and $q$ of $S$. Without loss of generality we assume that the line, $L$, through $p$ and $q$ is horizontal and that the $x$-coordinate of $p$ is less than the $x$-coordinate of $q$. The following lemma gives us a bound on DT $(p, q)$.

Lemma 1. Let $C$ be a circle whose boundary passes through $p$ and $q$, with center $O$ and radius $r$, and let $\theta$ be the upper angle $p O q$. If no point of $S$ lies inside $C$ below $L$, then $\mathrm{DT}(p, q) \leq r \theta(i . e ., \mathrm{DT}(p, q) \leq$ the length of the upper arc of $C$ from $p$ to $q$ ).

Proof. If no point of $S$ lies inside $C$, then DT $(p, q)=d(p, q) \leq r \theta$ for any such circle $C$ and we are done. Otherwise, let $t$ be the point in $S$ that lies inside $C$ such that $t$ lies on the boundary of the circle $D$, whose boundary passes through $p$ and $q$ such that no point in $S$ lies inside $D$ above the line $L$. Refer to Fig. 1(a) and (b). Let $C_{1}$ be the circle, with radius $r_{1}$, that passes through $p$ and $t$, whose centre $O_{1}$ lies on the segment $p O$, and let $C_{2}$ be the circle, with radius $r_{2}$, that passes through $t$ and $q$, whose centre $O_{2}$ lies on the segment $O q$. Let $a_{1}$ and $a_{2}$ be the respective intersection points of the circles $C_{1}$ and $C_{2}$ with the interior of the segment $p q$. Note that Fig. 1(a) shows a situation where $a_{2}$ is left of $a_{1}$, while Fig. 1(b) shows a situation where $a_{1}$ is left of $a_{2}$. Also let $C_{3}$ be the circle, with radius $r_{3}$, whose boundary passes through $a_{1}$ and $a_{2}$ and whose center, $O_{3}$, lies at the intersection of the line through $O_{1}$ and $a_{1}$ and the line through $O_{2}$ and $a_{2}$.

Using elementary geometry the following facts can be easily verified.

Fact 1 . No point of $S$ lies inside $C_{1}$ below the line through $p$ and $t$. Likewise, no point of $S$ lies inside $C_{2}$ below the line through $t$ and $q$.

Fact 2. Triangles $p O q, p O_{1} a_{1}, a_{2} O_{2} q$, and $a_{2} O_{3} a_{1}$ are all similar isoceles triangles with two equal base angles which we call $\varphi$. Thus the upper angles $p O q, p O_{1} a_{1}$, and $a_{2} O_{2} q$ are all equal to $\theta$. If $a_{2}$ is left of $a_{1}$, then the upper angle $a_{2} O_{3} a_{1}$ is also equal to $\theta$.

Fact 3.

$$
\cos \varphi=\frac{d\left(p, a_{1}\right)}{2 r_{1}}=\frac{d\left(a_{2}, q\right)}{2 r_{2}}=\frac{d\left(a_{2}, a_{1}\right)}{2 r_{3}}=\frac{d(p, q)}{2 r} .
$$

To prove the lemma, consider transforming $C$ downward into the circle $C^{\prime}$, with radius $r^{\prime}$ and center $O^{\prime}$, that passes through $p$ and $q$ and a third point $z$ of $S$ below $L$, maintaining the property that no point of $S$ lies inside $C^{\prime}$ below $L$. If we let $\theta^{\prime}$ be the upper angle $p O^{\prime} q$, then clearly $r^{\prime} \theta^{\prime} \leq r \theta$. Thus to prove the lemma for a circle $C$ it suffices to prove it for the corresponding circle $C^{\prime}$. If there is no such point $z$ below $L$, then the segment $p q$ forms part of the convex hull of $S$ and DT $(p, q)=d(p, q) \leq r \theta$ as required. Thus for the rest of the proof we assume that circle $C$ has been transformed to pass through a third point $z$ of $S$ that lies below $L$. 


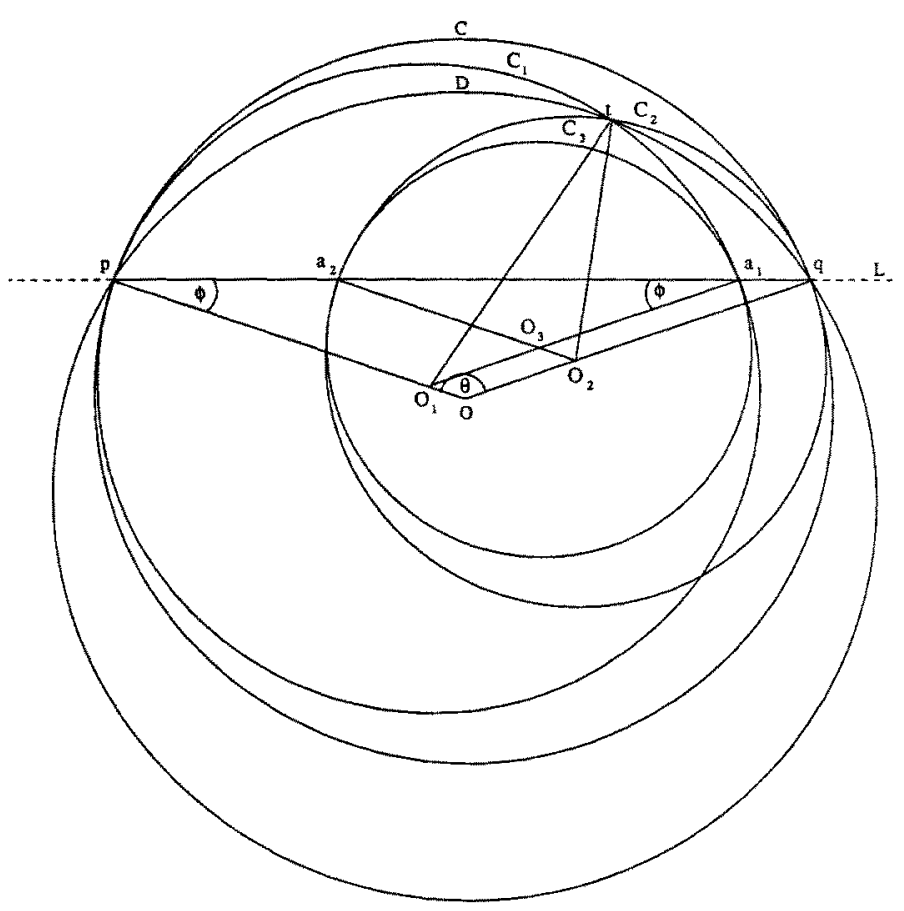

(a)

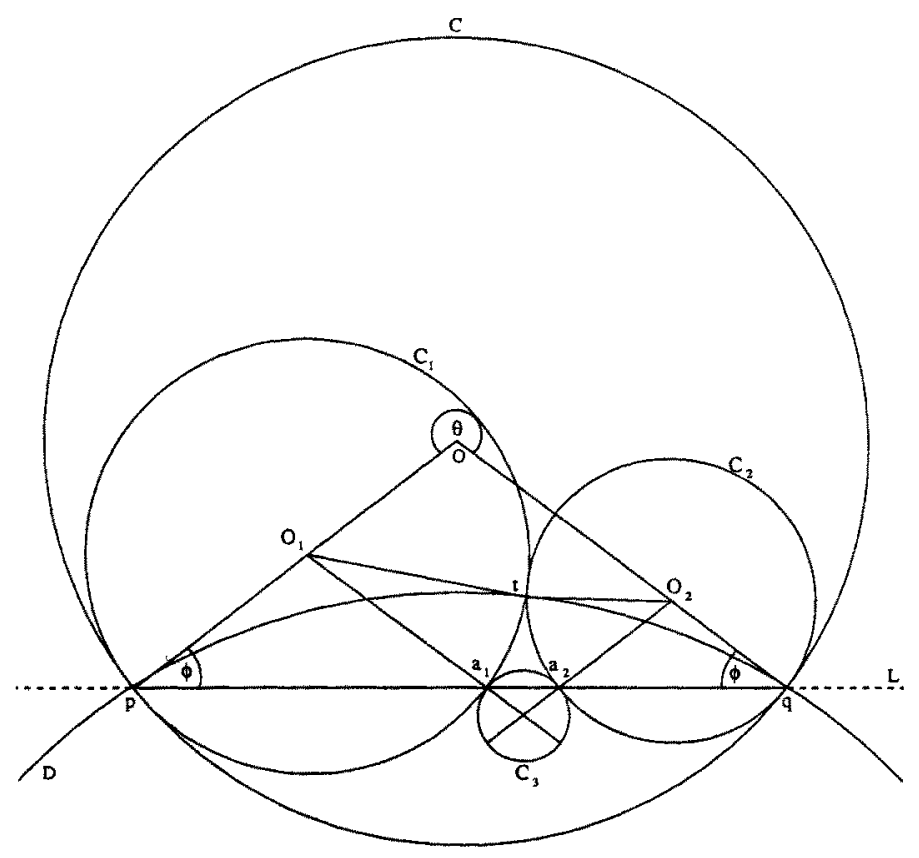

(b)

Fig. 1 
As there are only $O\left(n^{3}\right)$ possible transformed circles, we may proceed by induction on the rank of the angle $\theta$ in the transformed circle. As a base case consider the transformed circle, $C$, with the smallest possible angle $\theta$. If circle $C$ is not empty, then consider the circle $C_{1}$, defined earlier, that passes through $p$ and $t$. By Fact 1 we have that no point of $S$ lies inside $C_{1}$ below the line through $p$ and $t$ and by Fact 2 we have that the interior upper angle $\theta_{1}=$ ${ }_{p} O_{1} t<p O_{1} a_{1}=\theta$. But this contradicts the assumption that $C$ has the smallest possible interior upper angle $\theta$, thus $C$ must have been empty implying that $\mathrm{DT}(p, q)=d(p, q) \leq r \theta$ as required.

We may now consider a particular transformed circle $C$ and assume that the lemma holds for all other circles with a smaller interior upper angle. By Fact 2, we have $\theta_{1}=p O_{1} t<p O_{1} a_{1}=\theta$ and $\theta_{2}=t O_{2} q<a_{2} O_{2} q=\theta$. Thus Fact 1 and the inductive assumption imply that

$$
\mathrm{DT}(p, q) \leq \mathrm{DT}(p, t)+\mathrm{DT}(t, q) \leq r_{1} \theta_{1}+r_{2} \theta_{2} .
$$

Let $\bar{C}_{3}$ be the region that lies inside $C_{3}$ and above $L$. Let $\bar{C}_{12}$ be the region that lies inside $C_{1}$ and $C_{2}$ and above $L$. If $a_{2}$ is left of $a_{1}$, then $\bar{C}_{12}$ exists and properly contains $\bar{C}_{3}$. Since $\bar{C}_{3}$ and $\bar{C}_{12}$ are convex, we have $L\left(\bar{C}_{3}\right)<L\left(\bar{C}_{12}\right)$, where $L(X)$ is the length of the boundary of convex body $X[1, \mathrm{p}, 42]$. That is

$$
L\left(\bar{C}_{3}\right)=r_{3} \theta+d\left(a_{2}, a_{1}\right)<L\left(\bar{C}_{12}\right)=r_{1}\left(\theta-\theta_{1}\right)+r_{2}\left(\theta-\theta_{2}\right)+d\left(a_{2}, a_{1}\right) .
$$

Then from (1)

$$
\begin{aligned}
\operatorname{DT}(p, q) & \leq r_{1} \theta_{1}+r_{2} \theta_{2}=r_{1}\left(\theta-\left(\theta-\theta_{1}\right)\right)+r_{2}\left(\theta-\left(\theta-\theta_{2}\right)\right) \\
& =r_{1} \theta+r_{2} \theta-\left[r_{1}\left(\theta-\theta_{1}\right)+r_{2}\left(\theta-\theta_{2}\right)\right]<r_{1} \theta+r_{2} \theta-r_{3} \theta \\
& =\left(r_{1}+r_{2}-r_{3}\right) \theta
\end{aligned}
$$

by (2), and Fact 3 implies that

$$
\mathrm{DT}(p, q) \leq\left(r_{1}+r_{2}-r_{3}\right) \theta=\left(\frac{d\left(p, a_{1}\right)}{2 \cos \varphi}+\frac{d\left(a_{2}, q\right)}{2 \cos \varphi}-\frac{d\left(a_{2}, a_{1}\right)}{2 \cos \varphi}\right) \theta=\frac{d(p, q) \theta}{2 \cos \varphi}=r \theta .
$$

If $a_{2}$ is not left of $a_{1}$, then from (1) we have

$$
\mathrm{DT}(p, q) \leq r_{1} \theta_{1}+r_{2} \theta_{2} \leq r_{1} \theta+r_{2} \theta=\left(r_{1}+r_{2}\right) \theta
$$

and Fact 3 implies that

$$
\mathrm{DT}(p, q) \leq\left(r_{1}+r_{2}\right) \theta=\left(\frac{d\left(p, a_{1}\right)}{2 \cos \varphi}+\frac{d\left(a_{2}, q\right)}{2 \cos \varphi}\right) \theta \leq \frac{d(p, q) \theta}{2 \cos \varphi}=r \theta
$$

We are now able to prove the main theorem of this section. 
Theorem 1. Given a set $S$ of $N$ points in the plane, for any two points $p$ and $q$ in $S$

$$
\frac{\operatorname{DT}(p, q)}{d(p, q)} \leq \frac{2 \pi}{3 \cos \left(\frac{\pi}{6}\right)} \approx 2.42,
$$

independent of $S$ and $N$.

Proof. Let $C_{1}\left(C_{2}\right)$ be the circle that passes through $p$ and $q$ with centre $O_{1}\left(O_{2}\right)$ above (below) $L$ such that angles $q \mathrm{pO}_{1}\left(q \mathrm{pO}_{2}\right)$ and $\mathrm{pqO}_{1}\left(\mathrm{pqO}_{2}\right)$ equal $\pi / 6$. Let LUNE be the intersection of the interiors of $C_{1}$ and $C_{2}$. See Fig. 2.

If no points of $S$ lie inside LUNE, then $C_{1}$ is empty below $L$ and the lemma implies that DT $(p, q) \leq r_{1} \theta_{1}$ where $r_{1}$ is the radius of $C_{1}$ and $\theta_{1}$ is the upper angle $p O_{1} q$. We have $\theta_{1}=2 \pi-(\pi-2(\pi / 6))=\frac{4}{3} \pi$ and also

$$
r_{1}=\frac{d(p, q)}{2 \cos (\pi / 6)},
$$

thus

$$
\mathrm{DT}(p, q) \leq \frac{2 \pi}{3 \cos (\pi / 6)} d(p, q)
$$

as required.

It remains to consider the case where there exists a point of $S$ that lies inside LUNE. Without loss of generality we need only consider the set of points $S^{\prime} \subseteq S$ that lie inside LUNE and closer to $p$ than $q$. Let $\mathscr{C}$ be the set of circles that pass through point $p$ whose centers lie on the segment $p O_{1}$. Without loss of generality we may assume that there exists a point $t$ of $S^{\prime}$ that does not lie below $L$, such that the intersection of LUNE and the circle $C_{3} \in \mathscr{C}$ that passes through $t$ contains no point of $S$. Refer to Fig. 2. Let $\theta$ be the angle qpt and let $\varphi$ be the angle pqt. Let $c$ be the distance from $t$ to the closest point $t^{\prime}$ on the line $L$. Let $\alpha$ be the upper angle $\mathrm{pO}_{3} t$ and let $r$ be the radius of $C_{3}$.

We now proceed by induction on the rank of $d(p, q)$ with respect to pairs of point $p$ and $q$ in $S$. If $p$ and $q$ are the two closest points in $S$, then DT $(p, q)=d(p, q)$, as the segment $p q$ forms part of the Delaunay triangulation of $S[6]$.

As an inductive step consider the $i$ th closest pair $p$ and $q$ and assume that for all closer pairs the theorem holds. We have

$$
\mathrm{DT}(p, q) \leq \mathrm{DT}(p, t)+\mathrm{DT}(t, q) .
$$

Since $C_{3}$ is empty below the line through $p$ and $t$, Lemma 1 implies that $\mathrm{DT}(p, t) \leq \alpha r$. From the inductive assumption we have

$$
\mathrm{DT}(t, q) \leq \frac{2 \pi}{3 \cos (\pi / 6)} d(t, q) .
$$




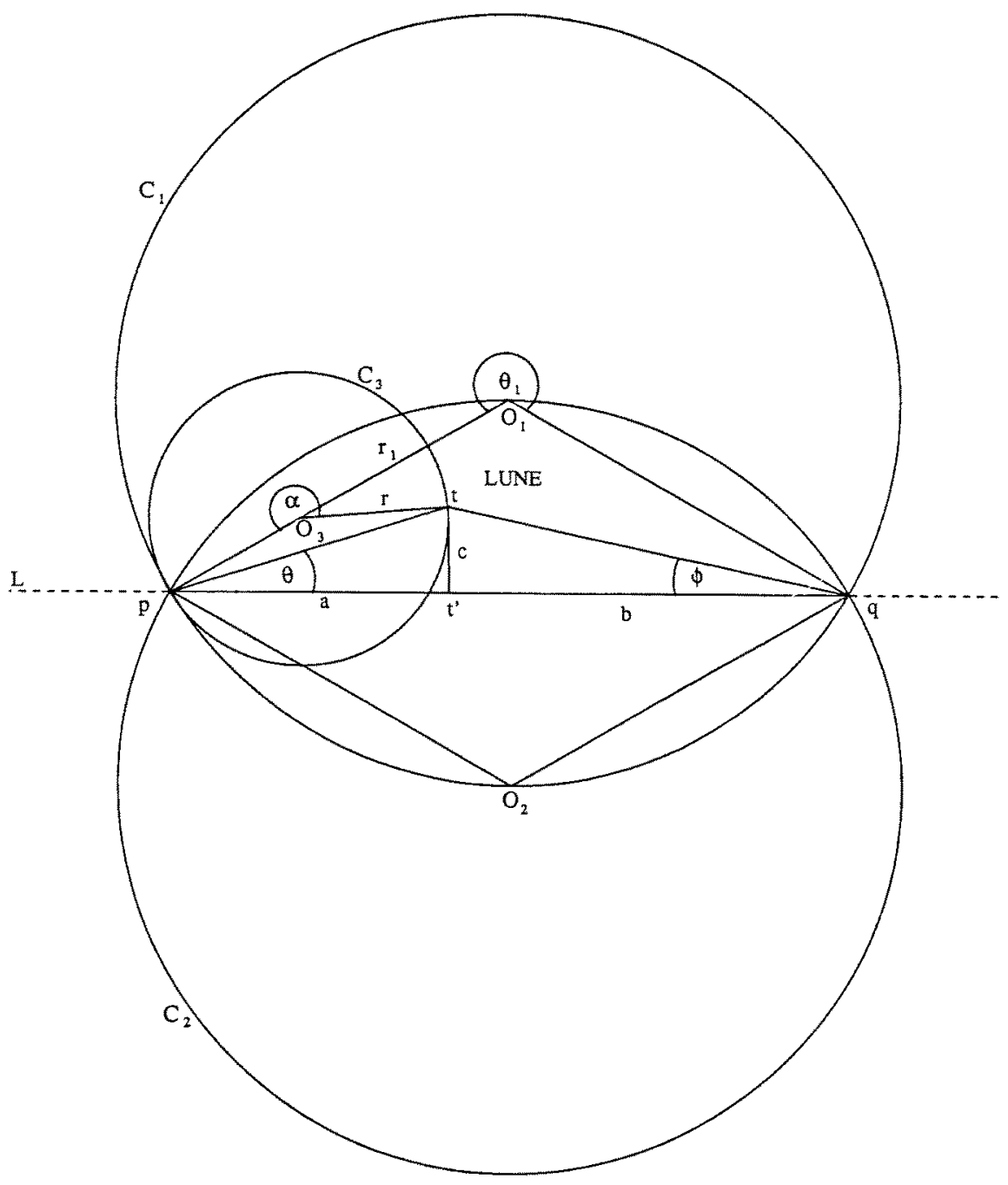

Fig. 2

Thus

$$
\mathrm{DT}(p, q) \leq \alpha r+\frac{2 \pi d(t, q)}{3 \cos (\pi / 6)}
$$

Consider the case in which $t$ lies on $L$. We have $\alpha=\frac{4}{3} \pi$ and $r=d(p, t) /(2 \cos (\pi / 6))$ therefore from (3) we have

$$
\mathrm{DT}(p, q) \leq \frac{2 \pi}{3 \cos (\pi / 6)}[d(p, t)+d(t, q)]=\frac{2 \pi}{3 \cos (\pi / 6)} d(p, q)
$$

as required. 
It remains to consider the case where $t$ lies above $L$. Using elementary geometry we have $\alpha=2 \pi-(\pi-2(\pi / 6-\theta))=\frac{4}{3} \pi-2 \theta$ and, since $d(p, t)=c / \sin \theta$, using the sine law we have

$$
r=\frac{c \sin (\pi / 6-\theta)}{\sin \left(\frac{2}{3} \pi+2 \theta\right) \sin \theta}=\frac{c}{2 \sin (\pi / 3+\theta) \sin \theta} .
$$

Since $d(t, q)=c / \sin \varphi$ we can substitute into (3) to get

$$
\mathrm{DT}(p, q) \leq\left(\frac{2}{3} \pi-\theta\right) \frac{c}{\sin (\pi / 3+\theta) \sin \theta}+\frac{2 \pi c}{3 \cos (\pi / 6) \sin \varphi} .
$$

Let $a$ be the distance from $p$ to $t^{\prime}$ and let $b$ be the distance from $t^{\prime}$ to $q$. Then

$$
d(p, q)=a+b=\frac{c}{\tan \theta}+\frac{c}{\tan \varphi},
$$

therefore

$$
\begin{aligned}
\frac{\mathrm{DT}(p, q)}{d(p, q)} & \leq \frac{\left(\frac{2}{3} \pi-\theta\right) /(\sin (\pi / 3+\theta) \sin \theta)+2 \pi /(3 \cos (\pi / 6) \sin \varphi)}{1 / \tan \theta+1 / \tan \varphi} \\
& =\left[\frac{\frac{2}{3} \pi-\theta}{\sin (\pi / 3+\theta) \sin \theta}+\frac{2 \pi}{3 \cos (\pi / 6) \sin \varphi}\right] \frac{\sin \theta \sin \varphi}{\cos \theta \sin \varphi+\cos \varphi \sin \theta} \\
& =\left(\frac{2}{3} \pi-\theta\right) \frac{\sin \varphi}{\sin (\pi / 3+\theta) \sin (\varphi+\theta)}+\frac{2 \pi \sin \theta}{3 \cos (\pi / 6) \sin (\varphi+\theta)}=f(\theta, \varphi)
\end{aligned}
$$

Since $t$ lies inside LUNE above $L$ and closer to $p$ than $q$ we have $\varphi>0, \theta \geq \varphi$, and $\theta+\varphi<\pi / 3$. With these contraints it can be shown that $\partial f / \partial \theta \neq 0$ if $\theta \geq \pi / 10$ and $\partial f / \partial \varphi \neq 0$ if $\theta \leq \pi / 10$. We thus have that $f(\theta, \varphi)$ achieves its maximum value on the boundary. There are three boundary conditions to check.

As $\varphi$ approaches, $0, \sin \varphi$ approaches 0 and $\sin (\varphi+\theta)$ approaches $\sin (\theta)$ thus

$$
\lim _{\varphi \rightarrow 0} f(\theta, \varphi)=\frac{2 \pi}{3 \cos (\pi / 6)} .
$$

As $\varphi$ approaches $\theta, f(\theta, \varphi)$ approaches

$$
\begin{aligned}
g(\theta) & =\left(\frac{2 \pi}{3}-\theta\right) \frac{\sin \theta}{\sin (\pi / 3+\theta) \sin (2 \theta)}+\frac{2 \pi \sin \theta}{3 \cos (\pi / 6) \sin 2 \theta} \\
& =\frac{2 \pi / 3-\theta}{\sin (\pi / 3+\theta) 2 \cos \theta}+\frac{\pi}{3 \cos (\pi / 6) \cos \theta},
\end{aligned}
$$


where $0<\theta<\pi / 6$. Using calculus it can be shown that $g(\theta)$ has one local minimum and no local maximum when $0<\theta<\pi / 6$, thus $g(\theta)$ must attain its maximum value as $\theta$ approaches either 0 or $\pi / 6$ :

$$
\lim _{\theta \rightarrow 0} g(\theta)=\frac{\pi}{3 \sin (\pi / 3)}+\frac{\pi}{3 \cos (\pi / 6)}=\frac{2 \pi}{3 \cos (\pi / 6)}
$$

and

$$
\lim _{\theta \rightarrow \pi / 6} g(\theta)=\frac{\pi}{4 \cos (\pi / 6) \sin (\pi / 2)}+\frac{\pi}{3 \cos ^{2}(\pi / 6)} \approx 2.30
$$

As $\theta+\varphi$ approaches $\pi / 3, f(\theta, \varphi)$ approaches

$$
h(\theta)=\left(\frac{2 \pi}{3}-\theta\right) \frac{\sin (\pi / 3-\theta)}{\sin (\pi / 3+\theta) \sin (\pi / 3)}+\frac{2 \pi \sin \theta}{3 \cos (\pi / 6) \sin (\pi / 3)},
$$

where $\pi / 6<\theta<\pi / 3$. It can be shown that $\partial h / \partial \theta \neq 0$ when $\pi / 6<\theta<\pi / 3$, thus $h(\theta)$ attains its maximum value either as $\theta$ approaches $\pi / 6$ or as $\theta$ approaches $\pi / 3$ :

$$
\lim _{\theta \rightarrow \pi / 6} h(\theta) \approx 2.30
$$

and

$$
\lim _{\theta \rightarrow \pi / 3} h(\theta)=\frac{2 \pi}{3 \cos (\pi / 6)}
$$

Thus we have

$$
\frac{\mathrm{DT}(p, q)}{d(p, q)} \leq \underset{0<\varphi \leq \theta<\pi / 3-\varphi}{f(\theta, \varphi)} \leq \frac{2 \pi}{3 \cos (\pi / 6)}
$$

as required.

\section{Fixed-Angle $\theta$-Graph}

Definition. Consider the shortest path from a point $p$ to a point $q$ in a Euclidean graph $G$. Let $r$ be the intermediate vertex adjacent to $p$ on this path. If the angle $q p r$ is small we might suspect that $G(p, q)$ is not much longer than $d(p, q)$, whereas if angle $q p r$ is large we might suspect a greater difference between $G(p, q)$ and $d(p, q)$. This intuition suggests that by defining a type of graph in which each point will be connected to a near neighbor in each of a variety of directions we may approximate the complete Euclidean graph. 


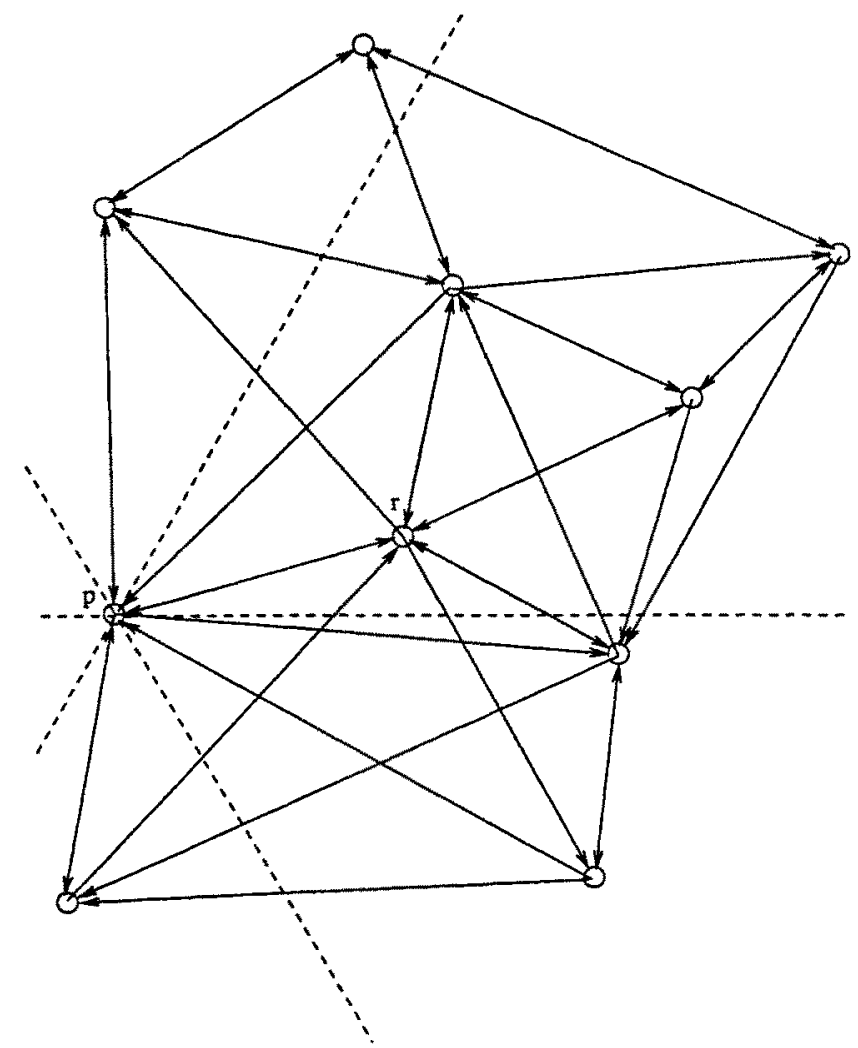

Fig. 3

Given a set $S$ of points in the plane we define the $\theta$-graph, for $\theta=2 \pi / k, k$ an integer constant such that $k>4$, to be the Euclidean graph, $\theta(S)$, whose edges are defined as follows. From a point $p \in S$, with $x$-coordinate $x(p)$ and $y$-coordinate $y(p)$, draw $k$ rays such that the rays form the angles $2 \pi(i-1) / k, i=1,2, \ldots, k$, respectively with the positive $x$-axis. In Fig. $3, k=6$ and the rays from point $p$ are drawn with dashed lines. These rays divide the plane into $k$ regions and we let $S_{i}(p), i=1,2, \ldots, k$, be the points $q \in S$ such that the angle $\varphi$ between the ray $\overrightarrow{p q}$ and the positive $x$-axis is such that $2 \pi(i-1) / k \leq \varphi<2 \pi i / k$. If $S_{1}(p)$ is not empty, then $p$ will be the source of a type 1 edge to a point $r \in S_{1}(p)$ such that $x(t)=\min \left\{x(q) \mid q \in S_{1}(p)\right\}$. If there are several candidates for $r$, the type 1 edge from $p$ will go to the point of minimum $y$ coordinate. In general, let $q^{\prime}$ be the perpendicular projection of a point $q \in S_{i}(p)$ on the ray from $p$ at angle $2 \pi(i-1) / k$. Then $p$ will be the source of a type $i$ edge destined to a point $r$ if and only if $r \in S_{i}(p)$, and for all other $q \in S_{i}(p)$ either $d\left(p, r^{\prime}\right)<d\left(p, q^{\prime}\right)$ or $d\left(p, r^{\prime}\right)=d\left(p, q^{\prime}\right)$ and $d\left(r, r^{\prime}\right)<d\left(q, q^{\prime}\right)$. Once the edges have been defined, the implied directions on the edges are ignored so that $\theta(S)$ is an undirected graph.

Note that a $\theta$-graph differs from a geographic neighbor graph [9] in that a point will not necessarily be connected to its nearest neighbor in each region. Also 
the $\theta$-graph is easier to compute. In the next section we present a simple $O(N \log N)$ plane sweep algorithm for constructing a $\theta$-graph. Until recently the best algorithm for the geographic neighbor graph required $O\left(N^{2-1 / 8} \log ^{2-1 / 8} N\right)$ time [9]. Recently, Wee et al. [8] presented a complex $O(N \log N)$ divide-and-conquer algorithm for this problem.

Algorithm. To construct the $\theta$-graph for a given point set the algorithm makes use of a separate plane sweep for each of the $k$ different types of edges.

Let us consider the plane sweep in which the type 1 edges are identified, as the other sweeps are performed similarly. During the performance of this plane sweep, three different orderings of the points are employed. These are the ordering by $x$-coordinate, the ordering by $y$-coordinate, and the $\gamma$ ordering by the projections of the points onto the oriented line $L$ through the origin at angle $2 \pi / k+\pi / 2$ with the positive $x$-axis which is oriented by the ray from the origin at angle $2 \pi / k+\pi / 2$ with the positive $x$-axis. For simplicity of presentation we assume that no two points have the same $x$-coordinate, the same $y$-coordinate, or the same projection onto the line $L$. Let $\gamma(p)$ be the rank of point $p$ in the $\gamma$ ordering.

The point set is swept in decreasing order of $y$-coordinate. As the sweep progresses a table $T$ of active points to which type 1 edges may be destined is maintained in $\gamma$ order. When a point $p$ is encountered on the sweep the following operations are performed:

1. Insert point $p$ into table $T$.

2. If $p$ has a predecessor $q$ in $T$, then report that $p q$ is a type 1 edge.

3. Repeat Forever

If $p$ has a successor $r$ in $T$, then

if $x(r)>x(p)$, then delete $r$ from $T$ else exit loop

else exit loop

Proof of Correctness. Here we prove that the above algorithm correctly computes the type 1 edges in $\theta(S)$. The proof that the other edges are correctly determined is analogous. Again for simplicity we assume that no two points have the same $x$-coordinate, the same $y$-coordinate, or the same projection onto the line $L$.

We first note that the table $T$ maintains the points in both $\gamma$ order and reverse $x$ order.

Lemma 2. Table $T$ is maintained so that if $s$ is the predecessor of $t$ in $T$, then $\gamma(s)<\gamma(t)$ and $x(s)>x(t)$.

Proof. We proceed by induction on the number of points encountered by the sweep. When a point $t$ is inserted into $T$ it is placed in $\gamma$ order. If successors of $t$ are then deleted, the table will remain in correct $\gamma$ order. It remains to show that correct reverse $x$ order is maintained in the neighborhood of $t$. If $s$ is the predecessor of $t$ in $T$, then, since $y(s)>y(t), \gamma(s)<\gamma(t)$, and $\theta=2 \pi / k \leq \pi / 2$, we have $x(s)>x(t)$. Also when step 3 of the loop is completed we have either $t$ has no successor in $T$ or, for the successor $r$ of $t$ in $T, x(r)<x(t)$. 
The next lemma shows that the points deleted in step 3 of the algorithm are not needed in further processing.

Lemma 3. If a point $r$ is deleted in step 3 of the algorithm, immediately after the insertion of a point $z$, then $r$ cannot be the destination of a type 1 edge whose source is a point $u$ with $y(u)<y(z)$.

Proof. Since $r$ is deleted by the insertion of point $z$, we know that $y(r)>y(z)$, $\gamma(r)>\gamma(z)$, and $x(r)>x(z)$. If a point $u$, with $y(u)<y(z)$, is to be the source of a type 1 edge destined to $r$ we must have that $\gamma(r)<\gamma(u)$. But we have $y(u)<y(z)$, $\gamma(z)<\gamma(r)<\gamma(u)$, and $x(z)<x(r)$, thus point $z$ prevents the formation of a type 1 edge from $u$ to $r$.

The final lemma of this section shows that type 1 edges are being correctly reported.

Lemma 4. When $p$ is inserted into table $T, p$ has predecessor $q$ in $T$ if and only if $p$ is the source of a type 1 edge to $q$ in the $\theta$-graph.

Proof. If $p$ has predecessor $q$ in $T$, then $y(p)<y(q)$ and $\gamma(p)>\gamma(q)$. By Lemma 2, no point $z$ exists in $T$ when $p$ is inserted, such that $y(p)<y(z), \gamma(z)<\gamma(p)$, and $x(z)<x(q)$. It remains to show that no point $r$, that has been deleted from $T$, exists, such that $\gamma(r)<\gamma(p)$ and $x(r)<x(q)$. Assume, to the contrary, that there are points that have been deleted from $T$ with $\gamma$ rank less than $\gamma(p)$ and $x$-coordinate less than $x(q)$. Let $r$ be such a point with minimum $y$-coordinate. The deletion of $r$ was caused by the insertion of a point $w$ with $y(p)<y(w)<y(r), \gamma(r)>\gamma(w)$, and $x(r)>x(w)$. If $\gamma(w)<\gamma(q)$, then the insertion of $w$ would have caused the deletion of $q$ contradicting the fact that $q$ is in $T$ when $p$ is inserted. If $\gamma(w)>\gamma(q)$, this contradicts the fact that $q$ is the predecessor of $p$ in $T$, since $\gamma(p)>\gamma(r)>\gamma(w)>\gamma(q)$.

If $p$ is the source of a type 1 edge to $q$ in the $\theta$-graph, then by definition we have $y(p)<y(q)$ and $\gamma(q)<\gamma(p)$. Then, by Lemma $3, q$ could not have been deleted from $T$ before $p$ was inserted. We thus have that when $p$ is inserted into $T, q$ exists in $T$ with $\gamma(q)<\gamma(p)$. If $q$ is not the immediate predecessor of $p$, then some point $z$ is such that $y(p)<y(z)$ and $\gamma(z)<\gamma(p)$. If $x(z)<x(q)$, then $p$ is not the source of a type 1 edge destined to $q$. If $x(z)>x(q)$, then either $\gamma(q)>\gamma(z)$ or $y(z)>y(q)$. In the former case, we have a contradiction to the assumption that $z$ is the immediate predecessor of $p$ in $T$. If $y(z)>y(q)$, then $z$ would have been deleted when $q$ was inserted since $\gamma(z)>\gamma(q), x(z)>x(q)$, and $T$ is maintained in both $\gamma$ order and reverse $x$ order.

We are now able to prove the main theorem of this section.

Theorem 2. The algorithm correctly computes the $\theta$-graph for a set $S$ of points in the plane in $O(N \log N)$ time, where $\theta=2 \pi / k$ and $k$ is an integer constant such that $k>4$. 
Proof. The correctness of the algorithm follows from Lemma 4. To verify the timing note that if the table of active points $T$ is maintained as a balanced binary tree (e.g., 2-3-tree), then the operations insert, delete, successor, and predecessor can be performed in $O(\log N)$ time. Thus each of the $k$ plane sweeps can be performed in $O(N \log N)$ time. Since $k$ is a constant, the total time required to form the $\theta$-graph is $O(N \log N)$.

\section{The Bound for $\theta$-Graphs}

Given a set $S$ of points in the plane and the $\theta$-graph $\theta(S)$, for $\theta=2 \pi / k, k$ an integer constant such that $k>8$, the purpose of this section is to show that the graph $\theta(S)$ closely approximates the complete Euclidean graph. We must thus show that the ratio $\theta(p, q) / d(p, q)$ is bounded by a small constant for any pair of points $p$ and $q$ in $S$. The shortest path from $p$ to $q$ in the graph $\theta(S)$ will pass through $m$, $0 \leq m \leq N-2$, intermediate points. The following lemma bounds the ratio $\theta(p, q) / d(p, q)$ with a function of $m$.

Lemma 5. If the shortest path from $p$ to $q$ in $\theta(S)$, for $\theta=2 \pi / k, k$ an integer constant such that $k>8$, passes through $m$ intermediate points, then

$$
\frac{\theta(p, q)}{d(p, q)} \leq \frac{1}{\cos \theta}\left(\frac{\tan ^{m} \theta-1}{\tan \theta-1}\right)+\tan ^{m} \theta
$$

Proof. Let $s_{i}$ be the $i$ th intermediate point on the path from $p$ to $q, 0 \leq i \leq m$, $s_{0}=p$. Let $\theta^{\prime}(p, q)$ be the length of the shortest path from $p$ to $q$ under the following restriction. If the origin is located at $s_{i}$ and $q$ is located so that the ray $\overrightarrow{s_{i} q}$ forms an angle $\varphi$ with the $x$-axis where $2 \pi(i-1) / k \leq \varphi<2 \pi i / k$, then the edge from $s_{i}$ to $s_{i+1}$ in the path from $p$ to $q$ is of type $i$. Clearly, $\theta(p, q) \leq \theta^{\prime}(p, q)$ thus the lemma will follow if we can prove the bound for $\theta^{\prime}(p, q)$.

We proceed by induction on $m$. In the base case, $m=0$, there are no intermediate points on the path from $p$ to $q$ and $\theta^{\prime}(p, q)=d(p, q)$.

As an inductive assumption we assume that if there are $m-1$ intermediate points on the shortest restricted path between two points $p$ and $q$, then

$$
\frac{\theta^{\prime}(p, q)}{d(p, q)} \leq \frac{1}{\cos \theta}\left(\frac{\tan ^{(m-1)} \theta-1}{\tan \theta-1}\right)+\tan ^{(m-1)} \theta
$$

As an inductive step we consider the case where there are $m$ intermediate points on the shortest restricted path between $p$ and $q$. Since there are $m-1$ intermediate points on the shortest restricted path from $s_{1}$ to $q$ in $\theta(s)$, the inductive assumption implies that

$$
\theta^{\prime}(p, q) \leq d\left(p, s_{1}\right)+d\left(s_{1}, q\right)\left[\frac{1}{\cos \theta}\left(\frac{\tan ^{(m-1)} \theta-1}{\tan \theta-1}\right)+\tan ^{(m-1)} \theta\right]
$$


If we let $\theta^{\prime \prime}(p, q)$ equal the right-hand side of $(*)$, the lemma will follow if we can prove the bound for $\theta^{\prime \prime}(p, q)$.

Let the origin be located at $p$. Without loss of generality let $q$ be located so that the angle $\varphi$ between the $x$-axis and the ray $\overrightarrow{p q}$ is such that $0 \leq \varphi<2 \pi / k$. We also then have that $s_{1}$ is located so that the angle $\alpha$ between the $x$-axis and the ray $\overrightarrow{p s}_{1}$ is such that $0 \leq \alpha<2 \pi / k$. To proceed further we need more information as to where $q$ and $s_{1}$ are located. The following claim allows us to restrict their locations.

Claim. The ratio $\theta^{\prime \prime}(p, q) / d(p, q)$ can attain its maximum when $q$ is located on the $x$-axis and $s_{1}$ is located to maximize the angle $\alpha$ such that $0 \leq \alpha<2 \pi / k$.

Proof of the Claim. We show how $q$ and $s_{1}$ can be moved to their desired locations without decreasing the value of the ratio $\theta^{\prime \prime}(p, q) / d(p, q)$. If we move $q$ and $s_{1}$ so that the values of $d\left(p, s_{1}\right)$ and $d\left(s_{1}, q\right)$ do not decrease and the value of $d(p, q)$ does not increase, then the value of the ratio $\theta^{\prime \prime}(p, q) / d(p, q)$ will not decrease.

If $s_{1}$ and $q$ are located such that angle $\alpha$ is less than angle $\varphi$, we then consider the polar coordinates of $s_{1}$ and $q,\left(\alpha, r_{s_{1}}\right)$ and $\left(\varphi, r_{q}\right)$ respectively. If we move $q$ to location $\left(\alpha, r_{q}\right)$ and $s_{1}$ to the location $\left(\varphi, r_{s_{1}}\right)$, we preserve the values $d\left(p, s_{1}\right)=r_{s_{1}}$, $d\left(s_{1}, q\right)$ and $d(p, q)=r_{q}$. We may thus hence forth assume that if $\varphi$ is the angle between the $x$-axis and the ray $\overrightarrow{p q}$ and $\alpha$ is the angle between the $x$-axis and the $\overrightarrow{p s}_{1}$, then $\alpha \geq \varphi$.

We now show how $q$ can be moved to the $x$-axis. To do this we rotate the segment $s_{1} q$ about $s_{1}$ until $q$ lies on the $x$-axis. This transformation clearly preserves the values $d\left(p, s_{1}\right)$ and $d\left(s_{1}, q\right)$. That $\alpha \geq \varphi$ ensures that the transformation does not increase the value of $d(p, q)$.

To complete the proof of the claim we need to show that we can move $s_{1}$ so that the angle $\alpha$ approaches $2 \pi / k$, without decreasing the ratio $\theta^{\prime \prime}(p, q) / d(p, q)$. To do this we move $s_{1}$ by increasing its $y$-coordinate as much as possible without violating the condition that $\alpha<2 \pi / k$. Since both $p$ and $q$ lie on the $x$-axis, this transformation will increase the values of $d\left(p, s_{1}\right)$ and $d\left(s_{1}, q\right)$, but will not affect the value of $d(p, q)$.

The claim implies that in $(*) 0 \leq d\left(p, s_{1}\right)<d(p, q) / \cos \theta$. Using elementary calculus it can be shown that $\theta^{\prime \prime}(p, q)$ will attain its maximum when $s_{1}$ is located so that $d\left(p, s_{1}\right)$ is maximized. In this situation we have $d\left(s_{1}, q\right)=d(p, q) \tan \theta$ thus

$$
\theta^{\prime \prime}(p, q) \leq \frac{d(p, q)}{\cos \theta}+d(p, q) \tan \theta\left[\frac{1}{\cos \theta}\left(\sum_{i=0}^{m-2} \tan ^{i} \theta\right)+\tan ^{(m-1)} \theta\right]
$$

Therefore the ratio

$$
\frac{\theta^{\prime \prime}(p, q)}{d(p, q)} \leq \frac{1}{\cos \theta}\left(\sum_{i=0}^{m-1} \tan ^{i} \theta\right)+\tan ^{m} \theta=\frac{1}{\cos \theta}\left(\frac{\tan ^{m} \theta-1}{\tan \theta-1}\right)+\tan ^{m} \theta
$$

as required. 
Note that since $\theta(p, q)$ may be strictly less than $\theta^{\prime \prime}(p, q)$, the above bound is not tight. It may not be possible to find a pair of points in a point set with $\theta(p, q)=\theta^{\prime \prime}(p, q)$ for $m>2$.

The bound given by the lemma is strictly increasing with $m$. By taking the limit as $m$ approaches infinity we have the following theorem.

Theorem 3. Given a set $S$ of $N$ points in the plane and the $\theta-\operatorname{graph} \theta(S)$, for $\theta=2 \pi / k$, $k$ an integer constant such that $k>8$, for any two points $p$ and $q$ in $S$

$$
\frac{\theta(p, q)}{d(p, q)} \leq \frac{1}{\cos \theta}\left(\frac{1}{1-\tan \theta}\right)
$$

independent of $S$ and $N$.

Given $\theta=2 \pi / k$, the following table illustrates the nature of the bound $B=$ $(1 / \cos \theta)(1 /(1-\tan \theta))$ :

\begin{tabular}{cccccccc}
\hline$k$ & 10 & 15 & 20 & 25 & 30 & 35 & 40 \\
$B$ & 4.52 & 1.97 & 1.56 & 1.39 & 1.30 & 1.24 & 1.20 \\
\hline
\end{tabular}

\section{Concluding Remarks}

We have shown that $\mathrm{DT}(S)$, the graph of the Delaunay triangulation of a point set $S,(2 \pi /(3 \cos (\pi / 6)) \approx 2.42)$-approximates the complete Euclidean graph, independent of $S$ and $N$. The obvious open problem is to improve this bound.

We also introduced fixed-angle $\theta$-graphs as a potential new tool in the investigation of problems involving shortest distances in the plane. For $\theta=2 \pi / k, k$ a constant such that $k>8$, we were able to develop an $O(N \log N)$-time algorithm for the construction of $\theta(S)$ and we showed that $\theta(S)((1 / \cos \theta)(1 /(1-\tan \theta)))-$ approximates the complete Euclidean graph, independent of $S$ and $N$. Note that as $k$ gets large this bound approaches one. It would be interesting to investigate $\theta$-graphs for higher-dimensional point sets.

Neither $\theta$-graphs nor Delaunay triangulations have bounded degree. Is there a graph with bounded degree which approximates the complete Euclidean graph?

\section{Acknowledgments}

This work was supported by the Natural Science and Engineering Research Council of Canada. The authors wish to thank R. Coulman for producing the figures. 


\section{References}

1. Benson, R., Euclidean Geometry and Convexity, McGraw-Hill, New York, 1966.

2. Chew, $P$., There is a planar graph almost as good as the complete graph, Proceedings of the Second Symposium on Computational Geometry, Yorktown Heights, NY, 1986, pp. 169-177.

3. Chew, $P$., There are planar graphs almost as good as the complete graph, Journal of Computer and System Sciences, 3(9) (1989), 205-219.

4. Dobkin, D., S. Friedman, and K. Supowit, Delaunay graphs are almost as good as complete graphs, Proceedings of the 28th Annual Symposium on Foundations of Computing, Los Angeles, CA, 1987, pp. 20-26.

5. Peleg, D., and A. A. Schaffer, Graph spanners, Journal of Graph Theory, 13(1) (1989), 99-116.

6. Preparata, F., and M. Shamos, Computational Geometry: an Introduction, Springer-Verlag, New York, 1985.

7. Sedgewick, R., and J. Vitter, Shortest paths in Euclidean graphs, Algorithmica, 1(1) (1986), 31-48.

8. Wee, Y. C., Chaiken, S., and Willard D. E., General metrics and angle restricted Voronoi diagrams, Presented at the First Canadian Conference on Computational Geometry, Montreal, August 1989.

9. Yao, A. C., On constructing minimum spanning trees in $k$-dimensional spaces and related problems, SIAM Journal on Computing, 11 (1982), 721-736.

Received June 27, 1989, and in revised form March 8, 1990. 